\title{
Vulvodynia in prepubertal girls: diagnosis
}

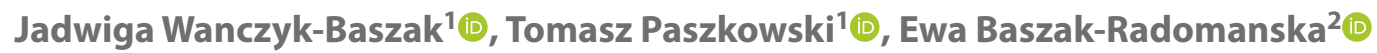 \\ ${ }^{1} 3^{\text {rd }}$ Chair and Department of Gynecology, Medical University of Lublin, Poland \\ ${ }^{2}$ Terpa, Lublin, Poland
}

\begin{abstract}
Objectives: To identify specific features of vulvodynia in prepubertal girls, highlight potential triggers and concomitant diseases, outline diagnostic criteria is neglected problem in adolescent gynecology.

Material and methods: A retrospective study, based on medical records of an outpatient clinic, a cohort of 54 vulvodynia cases was evaluated, aged 3-10 years, seen between January 2016 and July 2018.

Results: The study cohort presented with pain (61\%), sometimes aggravated at night, pruritus (44\%) and a range of other varied and unusual vulvar complaints (26\%). Concomitant diseases and/or psychological problems were present in $61 \%$ of cases. Overactive pelvic muscles accompanying symptoms like urological or gastrological problems were noted in half of children. Several potential triggers were identified in a third of the cases that were emotionally stressful to the children. From the commencement of symptoms, $93 \%$ of the girls have consulted more than one doctor with $43 \%$ seeing more than three doctors, without receiving a diagnosis of vulvodynia.

Conclusions: A diagnosis of vulvodynia needs to be considered in the absence of vulva pathology with wide range of vulvar pain, pruritus and discomfort. All persistent or recurrent vulvar discomfort must be taken into consideration as a vulvodynia symptom, also various non-specific, worrisome complaints. Comorbid urological and gastrological symptoms associated with overactive pelvic muscles should not be overlooked. Chronic pain can be triggered by the psychological distress in some prepubertal girls. Proper diagnosis may prevent long-term negative sequelae, what emphasizes the need for professional education of healthcare providers in adolescent vulvar pain and discomfort.
\end{abstract}

Key words: prepubertal; vulvar pain; vulvodynia

Ginekologia Polska 2022; 93, 11: 867-871

\section{INTRODUCTION}

Vulvodynia is suspected in adult women when vulvar pain or discomfort persists for more than three months and occurs in the absence of any evident pathology [1]. The same applies in the case of prepubertal girls. Vulvar skin disorders, as an identifiable cause of complaints need to be excluded [1-4]. Any vaginal discharge must be noted. Labial adhesions, when seen in prepubertal girls, are common and asymptomatic [5]. Foreign bodies in the vagina are not felt by the patient, unless they protrude to the vestibule, and these are rarely the cause of vulvar pain, vaginal discharge is present in such cases.

The prevalence of chronic vulvar pain in children is unknown, but in the general population vulvodynia is estimated to occur in $8.3-16 \%$ of adult women $[6,7]$. In keeping with recent nomenclature [1], vulvodynia in prepubertal girls, can be described as spontaneous or mixed, being ex- acerbated by vulvar touching (often for hygienic purposes) or pressure, when wearing tight clothes or playing, such as cycling. Unlike in adults, provoked vulvodynia is not as much of an issue in young girls except due to touch [8].

For affected children vulvar pain is mostly generalized. Because young girls are not familiar with their anatomy even localized pain is difficult for them to describe in any detail. Caregivers note that children usually complain of various symptoms but not pain per se $[4,8]$, and this can be misleading in terms of a diagnosis. Onset of pain is usually primary in nature, with potential triggers occasionally identified $[3,8]$. However, a constant form of vulvar pain is not typical in prepubertal girls. Children are often too occupied by daily activities to be conscious of persistent pain, hence it presents more as an intermittent or reactive pain.

Diagnosis, medical assessment and treatment of vulvodynia in young girls is comparable to adults $[2,7,9,10]$,

\footnotetext{
Corresponding author:

Ewa Baszak-Radomanska

Terpa, 34 Pogodna St, 20-333 Lublin, Poland

e-mail:ebarad@gmail.com
} 
though some differences need to be highlighted. Persistent vulvar pain or discomfort prevalence in children is unknown, though not rare, when moderate, recurrent rather than continuous complaints are considered. Given that healthcare practitioners are generally not familiar with vulvar pain in women, when pathology does not exist, it becomes an even a more neglected health issue in prepubertal girls.

\section{MATERIAL AND METHODS}

The study is based on a retrospective review of medical records from January 2016 to July 2018. A total of 54 cases were identified in the 3-10 years age group. All cases were seen in outpatient center specializing in vulvar diseases, including vulvodynia, but also with experience in child and adolescent gynecology. The study was approved by the appropriate IRB.

Cases were included if they satisfied the diagnostic criteria for vulvodynia, as outlined in the 2015 consensus statement by the International Society for the Study of Vulvovaginal Disease, International Society for the Study of Women's Sexual Health, and International Pelvic Pain Society [1].

The groups characteristics, including list of complains, duration, trigger factors, concomitant diseases and psychological problems, were all extracted from the medical records. Assessment, differential diagnosis and treatment was based on the center's clinical protocol and experience.

Diagnosis of vulvodynia was reached following the previously outlined assessment procedure [10]. Differential diagnosis of vulvar pain was made based on persistent or recurrent clinical symptoms, occurring over a period of more than three months, with no identifiable vulvovaginal pathology of vulvar and perianal skin, mucosa and discharge, upon pinworms exclusion in children.

The specific steps that were followed to reach a diagnosis, including:

1. All reported symptoms were noted in the patient history, as provided by parents and child: previous pathology, past treatments, reports of symptom triggers, past injuries (especially of a musculoskeletal nature), postural issues (postural alignment of hips, extremities and spine), prior surgery, prolonged use of medication, concomitant diseases, hygiene habits (use of herbs, hipbaths, vulvar skin applications, soaps, and other remedies). Also noted were any behavioral and psychological issues.

2. Vulvar and anal examination: specific focus on vulvar and perianal skin, mucosa and discharge (if present) for diagnosis other than vulvodynia. Parents were assured that in prepubertal girls, normal vestibular mucosa is more often red rather than pink, due to lack of estrogens [5]. Vaginal discharge was always noted in the presence of any foreign bodies (toilet paper or toy), these exams were possible when the child was asked to take deep breaths in and out, thus dilating the vaginal opening. Looking for causes of skin irritation, redness, small fissures, blisters like herpes simplex lesions, varicella and other ulcers (aphthous, Behcet or Crohn disease related), where present, these were identified. Signs of genital trauma, abuse [5] or any other injuries were noted. In any chronic vulvar pain cases the anus was also visually examined so as not to overlook possible anal fissure (which commonly appears at the 12 or 6 o'clock position - visualize a pelvic clock as reference). During all physical exams special attention and care was exercised to look for pinworms.

Cotton swab vulvar pressure as a Q-tip test and pelvic floor exam were not deemed necessary in young children and seem more applicable to exams of postmenarchal girls.

3. Lab tests: a diagnostic swab was taken from the vestibulum when discharge or skin erythema was noted, or reported by the mother, to exclude the presence of specific bacteria. Vulvovaginal yeast infections are rare in prepubertal girls [5]. Pinworms should also be excluded by a tap test. It is recommended that a small piece of transparent adhesive tape be used to take samples from skin around the anus, three days in a row. The tape as a specimen slide was sent to the laboratory to exclude presence of pinworms eggs. From a patient with urinary symptoms, a urine sample needs to be taken for analysis and culture.

Vulvodynia diagnosis is taken on the base of exclusion of any other visible or laboratory detectable pathology as the cause of symptoms.

Statistical analyses were performed using Statistica v. 9.1, provided by Statsoft Polska.

\section{RESULTS}

The age range of this study cohort of the 54 cases was 3-10 years of age, with a mean age of 5.8 years, constituting a prepubertal group of vulvodynia cases.

Vulvar pain and discomfort were perceived and experienced in different ways, with only two descriptors per patient being included in the summary data presented in Table 1.

The duration of symptoms was more than three months (up to maximum two years), often reported as remissions and recurrences. In many cases the exact time when the symptoms begun was not clear.

The diagnosis of vulvodynia in this cohort of prepubertal girls was made at the time of the first gynecological consultation at the vulvar specialist clinic. Prior to the diagnosis, 50 out of the 54 cases (93\%) consulted other doctors who included general practitioners, pediatricians, urologists, dermatologists and gynecologists. Since the onset of vul- 


\begin{tabular}{|c|c|c|}
\hline Symptom & $\begin{array}{l}\text { Number } \\
\text { of patients } \\
\text { (\% of cohort) }\end{array}$ & Pain characteristics/discription \\
\hline Pain & $\begin{array}{l}34(61 \%) \\
\text { patients }\end{array}$ & $\begin{array}{l}\text { Burning, twitching, soreness, } \\
\text { pulling/ drawing, tingling, } \\
\text { shooting, stabbing, twinge } \\
\text { - Often worse at nighttime }\end{array}$ \\
\hline Pruritus & $\begin{array}{l}24(44 \%) \\
\text { patients }\end{array}$ & $\begin{array}{l}\text { - Touching and scratching } \\
\text { - Rubbing }\end{array}$ \\
\hline $\begin{array}{l}\text { Uncommon } \\
\text { vulvar } \\
\text { complaints }\end{array}$ & $\begin{array}{l}14(26 \%) \\
\text { patients }\end{array}$ & $\begin{array}{l}\text { - "Something irritating" } \\
\text { - "Needles inside" } \\
\text { - "Hair tickling inside" } \\
\text { - "Air bubbles inside my bottom" } \\
\text { - "Poking and prodding" } \\
\text { - "Something moving in my } \\
\text { bottom" } \\
\text { - "Wet all the time" especially after } \\
\text { voiding, needing lots of toilet } \\
\text { paper to keep wiping } \\
\text { - "My underwear is always } \\
\text { wedging between buttock } \\
\text { cheeks" }\end{array}$ \\
\hline
\end{tabular}

vodynia symptoms 23 girls (43\%) had been seen by three or more healthcare practitioners. Due to recurrent bladder symptoms four of the children (7\%) were hospitalized. None of the 54 cases had received a diagnosis of vulvodynia prior to attending the clinic.

Concomitant diseases and psychological or emotional problems were noted in 33 cases $(61 \%)$, presenting with one or more associated comorbidities. Symptoms and comorbidities were divided into 4 groupings:

1. Symptoms associated with overactive pelvic muscles in 27 cases (50\%) which included:

a) Urological symptoms in 18 cases (33\%): with dysuria, frequency and urge, sometimes associated with incontinence (consistent with Overactive Bladder). This was often highlighted with accounts such as "she is so occupied by her play, that she keeps her bladder full and finally is dripping on the way to the toilet", or urinary tract infection symptoms (mostly without bacteriuria), difficulty initiating voiding and nocturnal enuresis.

b) Gastrological symptoms in 17 cases (31\%): with abdominal pain, irritable bowel syndrome, constipations, diarrhea, bowel incontinence (especially when not passing stool for a long time) and anal fissure.

2. Musculo-skeletal issues in five cases (9\%): presenting with postural defects and abdominal scar.

3. Other general variables in five cases (9\%): with allergies, recurrent respiratory tract infections, and headache.

4. Psychological and emotional factors in 11 girls (20\%): including previous participation in psychotherapy, dis- tress, anxiety, vulnerability, tics and childhood masturbation.

Some potential stressful triggers identified by parents in 18 cases (33\%) included: arrival of a newborn sibling, mother returning to work, a new babysitter, commencing kindergarten or school, and definite family problems.

\section{DISCUSSION}

Vulvodynia in prepubertal girls is a neglected healthcare problem. Comprehensive assessment of these condition is difficult, and diagnosis is often based on reported symptoms and professional experience. Among the wide spectrum of vulvar symptoms, persistent pain characterizes almost two thirds of all vulvodynia cases. Rating and character of pain was different, although sometimes aggravated at night with crying and waking up, what was the most distressing for the child and family. Almost half of vulvodynia girls seen complained of vulvar itch, and in these cases, it was essential that the presence of pinworms be excluded. A diagnosis of vulvodynia needs to be considered in the absence of vulvar pathology, although is often considered as vulvovaginal infection. The most symptomatic yeast vulvovaginal infections are seldom in children.

All persistent or recurrent vulvar discomfort must be taken into consideration as a vulvodynia symptom, also when children attempt to describe various non-specific, worrisome complaints (which was the case in every fourth of the affected girls), these included continuous wetness after voiding or bubbling-like sensations [11]. Vulvar pressure avoidance, pain on touching for hygienic purposes in children, this was considered as the equivalent of adult provoked vulvodynia symptoms $[3,4,8,11]$. The management of this disorder requires a highly skilled approach $[4,8,11,12]$.

The 2015 consensus terminology and classification statement noted comorbid pain syndromes as potential factors associated with vulvodynia [1]. Sixty one percent of prepubertal vulvodynia cases in this study presented with at least one comorbidity (physical or emotional).

Vulvodynia is associated with pelvic diaphragm dysfunction and should be considered as the part of regional anourogenital pain syndrome [13]. Concomitant complains of pelvic floor were seen in half of the prepubertal study population. Dysuria was noted in a third of the vulvodynia girls, and anal and bowel problems also presented in a third of study cohort. Urinary symptoms are the most reported associated symptoms $[11,13,14]$. On the basis of literature, dysuria, urinary frequency, incontinence (due to urge) were present in $80.6 \%$ of premenarchal and in $62.5 \%$ of postmenarchal adolescents with vulvodynia [11]. The incidences of urological symptoms were less often observed in this study consisting of 3-10 years old children. 
Pelvic floor dysfunction can be the consequence of other fascial and musculoskeletal defects in distant parts of the body in the case of vulvodynia. Abdominal scar tissue in early childhood is considered as a risk factor for chronic functional pain for reminder of life [15].

The cotton swab (Q-tip) test, first proposed by Friedrich in 1987, for the assessment of Vulvar Vestibulitis Syndrome [16] forms an important part of the diagnostic criteria for vulvodynia in adults. It consists of gently touching the vestibular hymen in approximately five points to identify areas of pain in vulvodynia patients. However, guided by the "First Do No Harm" principle [4], it is the view of the authors that it is not necessary to carry out a cotton swab vulvar touching test in the assessment of prepubertal girls. Other authors describe the procedure as useful in adolescences [3, 4, 8, 11, 14], but it may not be as appropriate for children. Children (and parents) are afraid of contact being made with the vulva, and it is difficult to draw information from little girls about sensitive areas that are painful. Based on the same principle, rectal pelvic floor muscles exams are not routinely performed. Diagnosis of vulvodynia is based on a clinical review and does not require a diagnostic biopsy [10].

Psychological predisposition and emotional states such as anxiety, distress and depression are all considered as contributor to functional chronic pain and vulvodynia $[3,4,8,12,17]$. This was shown to be the case in one fifth of the study cohort. The role of potential stressors needs to be assessed at an individual level. Early vulvodynia symptoms may suggest sexual abuse in the etiology of vulvar pain. Based on detailed history taking, external gynecological examination, abuse and violence must be excluded. In the literature no link has been established between physical or sexual abuse in the etiology of vulvodynia, though neglect, childhood chronic stress has been identified as predisposing factors $[11,18]$.

Some trigger factors such as life events that are noted in vulvodynia patients, may also be pertinent in children and adolescents $[3,8]$ : sexual and nonsexual physical contact, vaginal or vulvar infection, vulvar irritants in the form of herbal soap, wearing inappropriate cloths and use of antibiotics. Study authors consider several potential triggers that were evident in a third of the vulvodynia cases, that were clearly emotionally stressful events to the lives of children. Psychological distress plays crucial role in worsening of discomfort in chronic pain patients [12]. This includes numerous unnecessary consultations and medical procedures, in every age, prior to a diagnosis being established $[9,11,12]$. In the study cohort, from commencement of symptoms, almost all children have consulted at least one specialist and almost half seeing more than three doctors, without their vulvodynia being diagnosed. Chronic vulvar pain patients also seek medical consultations on account of concomitant diseases and/or psychological problems, often seen in prepubertal vulvodynia girls, highlighting the need for a holistic approach.

Vulvodynia diagnosis should be communicated to patients and their parents, explanation the disease and education is crucial $[4,9,11]$. Early diagnosis means commencement to treatment, proper educational, psychological stress reduction, physiotherapeutic intervention may prevent long-term negative physical and psychological sequelae [4], what emphasizes the need for professional education of healthcare providers in adolescent vulvar pain and discomfort.

Education on vulvar hygiene is essential for both children and parents, making them aware of possible allergens and irritants, and hence the recommendation for the use of natural emollients (such as coconut oil) is useful $[4,5,11]$. Vulvar pressure by some inappropriate cloths should be avoided, according to children's preferences.

Topical lidocaine treatment can be recommended in emergency situations, not as a long-term management [9].

According to literature, general pharmacological treatment in adolescent with severe vulvar pain, if administered with caution, may facilitate progress. In the case of adolescents, short-term therapy with tricyclic antidepressants (TCAs) can be used with persistent vulvar symptoms $[3,8,11$, 14], though more recent reviews do not recommend TCAs in vulvodynia $[4,9]$. Gabapentin has also been suggested, should be used with caution [3, 4]. Serotonin reuptake inhibitors (SSRIs), and rather serotonin and norepinephrine reuptake inhibitors (SNRI), are a preferred option for adolescents [3]. Duloxetine (SNRI) and pregabalin (anticonvulsant) may be advocated in adult cases, to decrease central and peripheral sensitization. With duloxetine there are no safety concerns in adolescents and children older than seven years for depressive disorders is accepted [19]. Pregabalin may be recommended in children, shown to be as safe and well tolerated in cases of epilepsy [20]. Any surgical vestibular approach (including partial hymenectomy) must be avoided in children.

Psychologic distress plays crucial role in worsening of discomfort in chronic pain patients. This includes numerous unnecessary consultations and medical procedures, in every age, prior to a diagnosis being established $[9,11,12]$. In the study cohort, from commencement of symptoms, $93 \%$ of children have consulted at least one specialist and $43 \%$ seeing more than three doctors, without their vulvodynia being diagnosed. Chronic vulvar pain patients also seek medical consultations on account of concomitant diseases and/or psychological problems (seen in $61 \%$ of prepubertal vulvodynia girls), highlighting the need for a holistic approach. Early diagnosis and proper intervention may prevent long-term negative physical and psychological sequelae [4]. 
Vulvar recurrence and chronic complaints in children are rare in the area of research and scientific publication. Thisstudy addresses many aspects of the problem, comprehensive diagnostic management is outlined, although study population is too small to elucidate various statistical relationships.

\section{CONCLUSIONS}

A diagnosis of vulvodynia needs to be considered in the absence of vulva pathology with wide range of vulvar discomfort. Pain characterizes almost two thirds and while half of all vulvodynia cases complained of vulvar itch. Pinworms and vulvovaginal infection should be excluded, although yeast infection is seldom in children. All persistent or recurrent vulvar discomfort must be taken into consideration as a vulvodynia symptom, also when children attempt to describe various non-specific, worrisome complaints. Comorbid urological and gastrological symptoms (in half of vulvodynia patients) associated with overactive pelvic muscles should not be overlooked. Chronic pain can be triggered by the psychological distress in some prepubertal girls $[11,18]$. Proper diagnosis may prevent long-term negative sequelae [4], what emphasizes the need for professional education of healthcare providers in adolescent vulvar pain and discomfort.

\section{Acknowledgment}

To Marek Jantos PhD for article edition support.

\section{Conflict of interest}

The authors report no conflict of interests.

\section{Funding}

Research based on own funds.

\section{REFERENCES}

1. Bornstein J, Goldstein AT, Stockdale CK, et al. Consensus vulvar pain terminology committee of the International Society for the Study of Vulvovaginal Disease (ISSVD), the International Society for the Study of Women's Sexual Health (ISSWSH), and the International Pelvic Pain Society (IPPS). 2015 ISSVD, ISSWSH, and IPPS consensus terminology and classification of persistent vulvar pain and vulvodynia. J Low Genit Tract Dis. 2016; 20(2): 126-130, doi: 10.1097/LGT.0000000000000190, indexed in Pubmed: 27002677.

2. Pukall CF, Goldstein AT, Bergeron S, et al. Vulvodynia: definition, prevalence, impact, and pathophysiological factors. J Sex Med. 2016;
13(3): 291-304, doi: 10.1016/j.jsxm.2015.12.021, indexed in Pubmed: 26944461.

3. Clare CA, Yeh J.Vulvodynia in adolescence: childhood vulvar pain syndromes. J Pediatr Adolesc Gynecol. 2011; 24(3): 110-115, doi: 10.1016/j. jpag.2010.08.009, indexed in Pubmed: 21601807.

4. Hersh JE. Vulvodynia in adolescents: presentation, diagnosis and treatment options. Curr Opin Obstet Gynecol. 2018; 30(5): 293-299, doi: 10.1097/GCO.0000000000000480, indexed in Pubmed: 30153128.

5. Simpson RC, Murphy R. Paediatric vulvar disease. Best Pract Res Clin Obstet Gynaecol. 2014; 28(7): 1028-1041, doi: 10.1016/j.bpobgyn.2014.07.004, indexed in Pubmed: 25134451.

6. Reed B, Harlow S, Sen A, et al. Prevalence and demographic characteristics of vulvodynia in a population-based sample. American Journal of Obstetrics and Gynecology. 2012; 206(2): 170.e1-170.e9, doi: 10.1016/j. ajog.2011.08.012

7. Harlow BL, Stewart EG. A population-based assessment of chronic unexplained vulvar pain: have we underestimated the prevalence of vulvodynia? J Am Med Womens Assoc (1972). 2003; 58(2): 82-88, indexed in Pubmed: 12744420.

8. Reed BD, Cantor LE. Vulvodynia in preadolescent girls. J Low Genit Tract Dis. 2008; 12(4): 257-261, doi: 10.1097/LGT.0b013e318168e73d, indexed in Pubmed: 18820538.

9. Goldstein AT, Pukall CF, Brown C, et al. Vulvodynia: assessment and treatment. J Sex Med. 2016; 13(4):572-590, doi: 10.1016/j.jsxm.2016.01.020, indexed in Pubmed: 27045258.

10. Nunns D, Mandal D, Byrne M, et al. British Society for the Study of Vulval Disease (BSSVD) Guideline Group. Guidelines for the management of vulvodynia. Br J Dermatol. 2010; 162(6): 1180-1185, doi: 10.1111/j.136 5-2133.2010.09684.x, indexed in Pubmed: 20331460.

11. Dunford A, Rampal D, Kielly $M$, et al. Vulval pain in pediatric and adolescent patients. J Pediatr Adolesc Gynecol. 2019; 32(4): 359-362, doi: 10.1016/j.jpag.2019.03.005, indexed in Pubmed: 30923024

12. Jantos M, Burns NR. Vulvodynia. Development of a psychosexual profile. J Reprod Med. 2007; 52(1): 63-71, indexed in Pubmed: 17286072.

13. Kennedy CM, Nygaard IE, Saftlas A, et al. Vulvar disease: a pelvic floor pain disorder? Am J Obstet Gynecol. 2005; 192(6): 1829-34; discussion 1834, doi: 10.1016/j.ajog.2004.12.059, indexed in Pubmed: 15970821.

14. Kielly $M$, Grover S. Vulvodynia in pre-menarchal girls: A case series. Journal of Pediatric and Adolescent Gynecology. 2016; 29(2): 206-207, doi: 10.1016/j.jpag.2016.01.110.

15. Stecco A, Stern R, Fantoni I, et al. Fascial disorders: implications for treatment. PM R. 2016; 8(2): 161-168, doi: 10.1016/j.pmrj.2015.06.006, indexed in Pubmed: 26079868.

16. Friedrich EG. Vulvar vestibulitis syndrome. J Reprod Med. 1987; 32: 110-14.

17. Lewandowski AS, Palermo TM, Stinson J, et al. Systematic review of family functioning in families of children and adolescents with chronic pain. J Pain. 2010; 11(11): 1027-1038, doi: 10.1016/j.jpain.2010.04.005, indexed in Pubmed: 21055709.

18. Dalton VK, Haefner HK, Reed BD, et al. Victimization in patients with vulvar dysesthesia/vestibulodynia. Is there an increased prevalence? J Reprod Med. 2002; 47(10): 829-834, indexed in Pubmed: 12418066.

19. Atkinson SD, Prakash A, Zhang Qi, et al. A double-blind efficacy and safety study of duloxetine flexible dosing in children and adolescents with major depressive disorder. J Child Adolesc Psychopharmacol. 2014; 24(4): 180-189, doi: 10.1089/cap.2013.0146, indexed in Pubmed: 24813026.

20. Mann D, Liu J, Chew ML, et al. Safety, tolerability, and pharmacokinetics of pregabalin in children with refractory partial seizures: a phase 1, randomized controlled study. Epilepsia. 2014; 55(12): 1934-1943, doi: 10.1111/epi.12830, indexed in Pubmed: 25377429. 